A most remarkable feature of HIV is its ability to replicate continuously and unrelentingly despite apparently strong antibody and cytotoxic T-lymphocyte (CTL) responses. Here, Ronald Desrosiers reviews the properties that allow the virus to evade ongoing immune responses and that present formidable obstacles to those seeking long-term control of HIV.

\title{
Strategies used by human immunodeficiency virus that allow persistent viral replication
}

That HIV replication is continuous and ongoing was not entirely appreciated during the early stages of theepidemic. In fact,

'Iatency', 'dormant period' and other such terms were used often in the 1980 s to refer to the long period of clinically inapparent infection that follows primary infection. Now, however, thereis no doubt that virus replication is continuous and ongoing during all stages of HIV infection. Viral dynamic studies have shown unequivocally that largenumbers of virus particles and infected cells are created and turned over every day ${ }^{1,2}$. Cellswithin thelymphoid tissues of SIV-infected macaque monkeys ${ }^{3,4}$ and HIVinfected people $e^{5}$ express viral products not only during primary infection but also during clinically inapparent and clinically apparent stages of infection long after the primary infection. This occurs despite the presence of readily measurableantiviral antibodies and antiviral CTLs. Antibodies that can bind viral proteins aretypically present at titers in thetensof thousands and antibodies specific for HIV envelope typically constitute $5 \%$ or more of the total IgG (ref. 6). Recent data indicate that $\mathrm{CD} 8^{+}$cells are important in at least limiting the amount of viral replication ${ }^{7}$. The numbers of $C D 8^{+} C T L$ effector cells to a single HIV epitope have been measured using precise tetramer technology and found to constitute as much as $1-5 \%$ of all CD 8 cells in the peripheral blood ${ }^{8}$. How can it be that cells are newly infected every day, express viral RNA and protein and make virus particles anew in the face of such high levels of antiviral antibodies and antiviral CTLs?

\section{Integration}

HIV and SIV, like other retroviruses, integrate DNA copies of their genetic information into the host cell genome. Expression of the viral genome in $\mathrm{CD}^{+} \mathrm{T}$ lymphocytes depends on the state of activation of the infected cells. Activation of selected populations of CD4 ${ }^{+}$Tlymphocytesisa normal responseto therecognition of foreign antigen. Many $\mathrm{CD}^{+} \mathrm{T}$ lymphocytes in the body are not activated, or areonly minimally activated, and arenot ideal substrates for the replication of HIV and SIV. Cellular activation increases, among other things, nucleotide pool sizes and the concentration of specific transcription factors. Without a proper state of cellu- lar activation, integrated proviral DNA may not be substantially expressed, essentially laying dormant until cellular activation can induce viral gene expression at a later time. Thus, al though virus replication is continuous and ongoing at all times after primary infection, thisdoes not mean that all infected cellsarein the process of active virus production or reproduction. In fact, the numbers of cells containing proviral DNA arefar greater than thenumbers of cells that can be detected actively expressing viral RNA or protein ${ }^{5}$. This dormancy may be an important factor in the retention of residual levels of infectious cellslong after continuous, highly active anti-retroviral therapy (HAART) ${ }^{9}$.

Latency may facilitateviral persistence by allowing long-term storage of viral genetic information, essentially archiving genes and epitopesthat can reemerge at a later date to test immune surveillance. However, it is important to emphasize that the ability of HIV to integrate its genetic information and the capacity for integrated proviral DNA to remain dormant in cells for prolonged periods cannot explain the persistent viral replication. Other factors must be involved in allowing HIV to evade ongoing immune responses.

\section{Emergence of antigenic variants}

The best estimate for the error rate of HIV reverse transcriptase is about 1 in 10,000 nucleotides $^{10}$. This means that each newly infected cell will on average introduceabout onemutation into the viral genome. Given that millions of newly infected cells are generated each day, the potential for genetic variation is enormous. Any genetic change that results in a growth advantage will be quickly selected for. In particular, any mutation that confers resistance to existing antiviral immunity without having debilitating effectson theinherent ability of virusto replicate will havean obvious selective advantage. In fact, mutations that reduce inherent replicative capacity may still be selected in vivo if they increase the effective replicative capacity sufficiently by avoidance of immune recognition. Selective advantage may al so result from other pressures: the ability to replicate in certain types of cells or tissues in which the virus may reside or the ability to replicate in a new host with a different genotypic background. What is remark- 
able about HIV, SIV and other lentiviruses is not just the rate at which they can accumulate mutations but al so their ability to toleratethesemutations, particularly in theenvelopesurface protein ${ }^{11}$. Amino acid changes becomefixed in the viral-encoded surfaceprotein population at a rateof about 15 per year $^{12}$ and individual envelope proteins of independent HIV-1 isolates may vary by as much as $35 \%$ in their amino acid sequence ${ }^{13}$.

The emergence of mutant forms of virus that result in escape from neutralizing antibody responses and escape from CTL responses during the course of infection of a single individual has been well documented. Monkeys infected with SIV derived from cloned DNA of defined sequence accumulated nucleotidechanges in gp120 envelope over time at a rate of about $1 \%$ per year ${ }^{12}$. Infected monkeys mounted antibody responses that could neutralize the incoming cloned virus, but the sequence variants that emerged were al so neutralization escape variants ${ }^{14}$. Escape from CTLs can occur by altering the ability of foreign peptide to be anchored in $\mathrm{MHC}$ or by al tering recognition by the T-cell receptor. Borrow et al. ${ }^{15}$ studied a patient with a strong, early, B44-restricted CTL response to an immunodominant epitope in Env. Genetic changesappeared in the predominant population of virus by $30-72$ days after primary infection, and these resulted in escape from recognition by theCTLs (ref. 15). Similar resultshavebeen described in six B27-positive patients; however, in these studies the time to the appearance of escape mutants was considerably longer ${ }^{16}$.

\section{Destruction of CD4 helper cell activity}

The dearth of anti-HIV CD4 hel per cell activity in HIV-infected people has been known for more than a decade ${ }^{17,18}$. However, the importance of this observation has only recently comeinto clearer focus $^{19}$. Individuals who seem to be controlling their HIV-1 infections remarkably well and who are long-term non-progressorsquite uniformly show strong, HIV-specific CD4 proliferative activity ${ }^{20}$. Theseindividualstypically maintain low or undetectableviral loads in the absence of anti-retroviral therapy. The strong HIV-specific CD4 proliferative activity in these unusual non-progessors contrasts with the absent or lower proliferative activity seen in typical progressors ${ }^{20,21}$.

CD4 cells respond to sites of infection in the body through specific, T cell receptor-mediated interactions with foreign peptideon MHC. Responding CD 4 cells become activated and facilitate CTL and antibody responses principally through the release of specific chemokines and cytokines. The picture that emerges is one of a battle during the initial weeks after HIV-1 infection between the virus trying to replicate in CD4 cells and CD4 cells trying to respond to sites of viral replication. Unfortunately for the host, these activated CD4 lymphocytes trying to respond to the infection are the ideal targets for replication of the virus. Most of the time, HIV ends up winning the battle, leaving the host without HIV-specific CD4 cells to provide help for the B-cell and CD8 CTL responses. Losing this essential battle early in the war also results in loss of the war, at least in the absence of anti-retroviral therapy. Only those individuals who for whatever reason are able to maintain substantial CD4 helper cell responses end up controlling HIV long-term. Infection by an attenuated virus may beonefactor that can shift the balance in favor of the host CD4 response ${ }^{22-24}$.

This emerging picture, still in need of more experimental verification, has enormously important implications for the future of drug treatment and vaccine development. Physicians are now facing serious difficulties in keeping their patients on HAART for 2-5 years or more. The 'holy grail' of drug treatment is to define conditions that will allow patients to discontinue HAART while still maintaining suppressed levels of HIV. This effort is now clearly focusing on regimens or treatments that will al low strong CD4 helper cell activity when HAART is discontinued. This emerging picture al so suggests that if a vaccine does not create a sterilizing barrier, it will likely need to inducestrong, HIV-specific CD4 hel per cell activity that can be present at the time of HIV exposure.

\section{Accessibility of envelope protein on virions}

HIV-infected peopleand SIV-infected rhesusmonkeysmakehigh levels of antibodies reactive with the viral-encoded envelope proteins gp120 and gp41. These antibodies react very well and with high affinity to soluble, monomeric envelop protein. However, they react poorly, not at all, or only with low affinity to thenative, oligomeric envelope protein as it exists on the surface of virions $\mathrm{s}^{25-28}$-an important observation generally underappreciated even in the AIDSresearch community. The inaccessibility of envelopeprotein on virions is especially true of primary isolates, whose difficulty to neutralize has been well documented ${ }^{28,29}$. The ability of antibody to neutral izeviral infectivity has been directly correlated with ability to bind to native, oligomeric protein on virions or on the surface of infected cells ${ }^{25,26}$. In contrast, most antibodies that can react with envelope protein do not neutralize and do not react appreciably with the oligomeric form. Antibodies that can bind virions and neutral ize infectivity typically do so only in a very strain-restricted manner. This inaccessibility of virus to antibodies occurs despite the presence of binding sites on the surface glycoprotein for two different receptors (CD4 and CCR5). Virions also seem to incorporate cellular proteinsthat make them resistant to complement-mediated virolysis ${ }^{30}$. Thus, although high levels of antibodies against env are produced during the course of infection, most of the antibody specificities seem to be ineffective.

An antibody may have difficulty accessing theen velopeprotein on the surface of virions at least in part because of the way the protein folds upon itself in oligomers ${ }^{31-34}$. Another contributing factor is the presence of extensive carbohydrate. In fact, approximately $50 \%$ of the mass of gp120 is carbohydrate, making it one of the most extensively glycosylated proteinson record. Mutated versions of SIVmac239 missing two $\mathrm{N}$-linked glycosylation sites within variableregion 1 (V1) were much more immunogenic (that is, better at eliciting neutral izing antibodies) and much moreantigenic (a more sensitivetarget for neutral ization) than the parental strain from which they were derived ${ }^{35}$. This was true for any pairwisecombination of the fourth, fifth and sixth glycosylation addition sites of the 24 sites present in gp120. Although a mutated virus missing five $\mathrm{N}$-linked sites in the $\mathrm{V} 1-\mathrm{V} 2$ regions replicated normally in monkeys for the first 2 weeks, it was effectively neutralized and controlled at low or undetectable levels for morethan 2 years $^{35}$. Elimination of selected carbohydrate attachment sites is therefore a promising new avenue for improvement of antibody responses by candidate vaccines. Another is the ability of envexpressing cells locked into a 'fusion-competent' state to induce broadly neutralizing antibodies ${ }^{36}$.

Other RNA viruses with error-prone polymerases, such as poliovirus, seem nowhere near as difficult to neutralize and nowhere near as malleable as HIV. The basis for these differences may lie in the inherent strategies used by persisting and nonpersisting viruses. Any virus must depend for its survival on the ability to spread through thepopulation. For anon-persisting virus such as poliovirus, transmission is limited to the several weeks after thetime of primary infection. Resistance to antibodies, once they appear, may not be as important to such a virus. Instead, it 
is important to replicate maximally for the brief period in order to maximize the likelihood for transmission. HIV, SIV and other lentiviruses, in contrast, help ensuretheir transmission by greatly lengthening the period during which transmission can occur. Thus, these viruses may sacrifice inherent replicative capacity in theshort term to allow persistent viral replication in thelongterm.

\section{Downregulation of $\mathrm{MHC}$}

Many viruses seem to have evolved strategies to minimize antigen presentation to the host immunesystem (review, ref. 37). For example, transforming strains of adenovirus encodea protein (E3) that prevents $\mathrm{MHC}$ class I molecules from reaching the cell surface $^{38,39}$. Herpessimplex virusencodesa protein (ICP47) that inhibits the peptide transporter TAP (ref. 40); TAP is needed to transport peptides to the MHC molecules that will present them as foreign.

The N ef proteins of HIV and SIV interact with and modify the endocytic and sorting pathways of thecell ${ }^{41}$. Oneresult of this interaction is the downregulation of $\mathrm{MHC}$ class I molecules from the surface of the cell, first shown by Schwartz et al ${ }^{42}$ Target cellsfrom which $\mathrm{MHC}$ class 1 molecules have been internalized by expression of Nef become resistant to the cytolytic activity of MHCrestricted CTLs (ref. 43). Although it is easy to see how this could contribute to immune evasion by the virus, the physiologic relevance of the observation is not entirely clear. MHC downregulation occurs at the highest level sof $\mathrm{Nef}$ expression ${ }^{43}$ and no onehas shown whether infected cells as they exist in the host have downregulated their $\mathrm{MHC}$ class I molecules.

\section{Conclusion}

Further advancement of therapeutic regimens and development of effective vaccines against HIV must deal with the strategies of immune evasion summarized in thisreview. Deal ing with any one alone would be difficult. Dealing with all of them will be heroic. The most promising tactics may be those that take advantage of the natural immune control that can sometimes occur. Drug regimens that facilitate the ability of the immune response to do its job are certainly the wave of the future. For vaccine development, immunologic memory induced by non-persisting antigensand vectorsisnot likely to be sufficient. Futurevaccine research will probably need to focus more on vehicles that allow persistence of antigen or persistence of antigen expression.

1. Wei, X. et al. Viral dynamics in human immunodeficiency virustype 1 infection. Nature 373, 117-122 (1995).

2. Ho, D.D. et al. Rapid turnover of plasma virions and CD4 lymphocytes in HIV-1 infection. Nature 373, 123-126 (1995).

3. Wyand, M.S. et al. Cellular localization of simian immunodeficiency virus in lymphoid tissues. II. In situ hybridization. American Journal of Pathology 134, 385-393 (1989).

4. Ringler, D.J. et al. Cellular localization of simian immunodeficiency virus in lymphoid tissues. I. Immunohistochemistry and electron microscopy. Am. J. Pathol. 134, 373-383 (1989).

5. Pantaleo, G. et al. HIV infection is active and progressive in lymphoid tissue during the clinically latent stage of disease. Nature 362, 355-358 (1993)

6. Binley, J.M. et al. Differential Regulation of the antibody responsesto gag and env proteins of human immunodeficiency virus Type 1. Virology 71, 2799-2809 (1997).

7. Schmitz, J.E. et al. Control of viremia in simian immunodeficiency virus infection by CD8+lymphocytes. Science 283, 857-860 (1999).

8. Ogg, G.S. et al. Quantitation of HIV-1-specific cytotoxic T lymphocytes and plasma load of viral RNA. Science 279, 2103-2106 (1998)

9. Finzi, D. et al. Latent infection of CD4+T cells provides a mechanism for lifelong persistence of HIV-1, even in patients on effective combination therapy. $N$ ature $M$ ed. 5, 512-517 (1999).

10. Mansky, L.M. \& Temin, H.M. Lower in vivo mutation rate of human immunodeficiency virus type 1 than that predicted from the fidelity of purified reverse transcriptase. J. Virol. 69, 5087-5094 (1995).

11. Coffin, J.M. Genetic variation in AIDS viruses. Cell 46, 1-4 (1986).

12. Bums, D.P.W. \& Desrosiers, R.C. Selection of genetic variants of simian immunodeficiency virus in persistently infected rhesus monkeys. J. Virol. 65, 1843-1854 (1991).
13. Myers, G. et al. in Human Retroviruses and AIDS 1995 (Los Alamos National Laboratory, Los Alamos, New Mexico, 1995).

14. Bums, D.P.W., Collignon, C. \& Desrosiers, R.C. Simian immunodeficiency virusmutants resistant to serum neutralization arise during persistent infection of rhesus monkeys. J. Virol. 67, 4104-4113 (1993).

15. Borrow, P. et al. Antiviral pressure exerted by HIV-1-specific cytotoxic T lymphocytes (CTLS) during primary infection demonstrated by rapid selection of CTL escape virus. Nature Med. 3, 205-211 (1997).

16. Goulder, P.J.R. et al. Late escape from an immunodominant cytotoxic T-lymphocyte response associated with progression to AIDS. Nature M ed. 3, 212-217 (1997).

17. Lane, H.C. et al. Qualitative analysis of immune function in patients with the acquired immunodeficiency syndrome. Evidence for a selective defect in soluble antigen recognition. N. Engl. J. Med. 313, 79 (1985).

18. Clerici, M. et al. Interleukin-2 production used to detect antigenic peptide recognition by T-helper lymphocytes from asymtomatic HIV-seropositive individuals. Nature 339, 383 (1989).

19. Rosenberg, E.S. \& Walker, B.D. HIV type 1-specific helperT cells: A critical host defense. AIDS Res. Hum. Retroviruses 14, S-143-S-147 (1998).

20. Rosenberg, E.S. et al. Vigorous HIV-1-specific CD4 ${ }^{+} \mathrm{T}$ cell responses associated with control of viremia. Science 278, 1447-1450 (1997).

21. Pitcher, C.J. et al. HIV-1-specific $\mathrm{CD}^{+} \mathrm{T}$ cells are detectable in most individuals with active HIV-1 infection, but decline with prolonged viral suppression. Nature M ed. 5, 518-525 (1999).

22. Greenough, T.C., Sullivan, J.L. \& Desrosiers, R.C. Declining CD4 T-cell countsin a person infected with nef-deleted HIV-1. N. Engl. J. Med. 340, 236-237 (1999).

23. Dyer, W.B. et al. Strong human immunodeficiency virus (HIV)-specific cytotoxic Tlymphocyte activity in Sydney blood bank cohort patients infected with nef-defective HIV type 1. J. Virol. 73, 436-443 (1999).

24. Gauduin, M.-C., Glickman, R.L., Ahmad, S., Yilma, T. \& Johnson, R.P. Characterization of SIV-specific CD4+Thelper proliferative responses in macaques immunized with live attenuated SIV. J. Med. Primatol. (in the press).

25. Fouts, T.R., Binley, J.M., Trkola, A., Robinson, J.E. \& Moore, J.P. Neutralization of the human immunodeficiency virus type 1 primary isolate JR-FL by human monoclonal antibodies correlates with antibody binding to the oligomeric form of the envelope glycoprotein complex. J. Virol. 71, 2779-2785 (1997).

26. Parren, P.W.H.I. et al. Neutralization of human immunodeficiency virustype 1 by antibody to gp120 is determined primarily by occupancy of sites on the virion irrespective of epitope specificity. J. Virol. 72, 3512-3519 (1998).

27. Sattentau, Q.J. \& Moore, J.P. Human immunodeficiency virus type 1 neutralization is determined by epitope exposure on the gp120 oligomer. J. Exp. Med. 182, 185-196 (1995).

28. Burton, D.R. A vaccine for HIV type 1: The antibody perspective. Proc. Natl. Acad. Sci. USA 94, 10018-10023 (1997).

29. Moore, J.P. et al. Primary isolates of human immunodeficiency virus type 1 are relatively resistant to neutralization by monoclonal antibodies to gp120, and their neutralization is not predicted by studies with monomeric gp120. J. Virol. 69, 101-109 (1995).

30. Takefman, D.M., Sullivan, B.L., Sha, B.E \& Spear, G.T. Mechanisms of resistance of HIV-1 primary isolates to complement-mediated lysis. Virology 246, 370-378 (1998).

31. Chan, D.C., Fass, D., Berger, J.M. \& Kim, P.S. Core structure of gp41 from the HIV envelope glycoprotein. Cell 89, 263-273 (1997).

32. Kwong, P.D., Wyatt, R., Robinson, J., Sweet, R.W. \& Sodroski, J. Structure of an HIV gp120 envelope glycoprotein in complex with the CD4 receptor and a neutralizing human antibody. Nature 393, 648-659 (1998).

33. Weissenhom, W., Dessen, A., Harrison, S.C., Skehel, J.J. \& Wiley, D.C. Atomic structure of the ectodomain form HIV-1 gp41. Nature 387, 426-430 (1997).

34. Wyatt, R et al. The antigenic structure of the HIV gp120 envelope glycoprotein. Nature 393, 705-711 (1998).

35. Reitter, J.N., Means, R.E. \& Desrosiers, R.C. A role for carbohydrates in immune evasion in AIDS. Nature Med. 4, 679-684 (1998).

36. LaCasse, R.A. et al. Fusion-competent vaccines: broad neutralization of primary isolates of HIV. Science 283, 357-362 (1999).

37. Ploegh, H.L. Viral strategies of immune evasion. Science 280, 248-253 (1998)

38. Andersson, M., Paabo, S., Nilsson, T. \& Peterson, P.A. Impaired intracellular transport of class $1 \mathrm{MHC}$ antigens as a possible means for adenoviruses to evade immune surveillance. Cell 43, 215-222 (1985).

39. Burgert, H.-G. \& Kvist, S. An adenovirustype 2 glycoprotein blocks cell surface expression of human histocompatibility class 1 antigens. Cell 41, 987-997 (1985).

40. York, I.A. et al. A cytosolic herpes simplex virus protein inhibits antigen presentation to CD8+T lymphocytes. Cell 77, 525-535 (1994).

41. Greenberg, M.E. et al. Co-localization of HIV-1 Nef with the AP-2 adaptor protein complex correlates with Nef-induced CD4 down-regulation. EM BO J 16, 6964-6976 (1997).

42. Schwartz, O., Marechal, V., LeGall, S., Lemonnier, F. \& Heard, J.-M. Endocytosis of major histocompatibility complex class I molecules is induced by the HIV-1 nef protein. Nature Med. 2, 338-342 (1996).

43. Collins, K.L., Chen, B.K., Kalams, S.A., Walker, B.D. \& Baltimore, D. HIV-1 Nef protein protects infected primary cells against killing by cytotoxic T lymphocytes. Nature 391, 397-401 (1998).

Harvard Medical School New England Regional Primate Research Center One Pine Hill Drive, Box 9102,

Southborough, Massachusetts 01772-9102, USA

Email: ronald_desrosiers@hms.harvard.edu 